\title{
Nanofibers Reinforced Polymer Composite Microstructures
}

\author{
A. Alubaidy, K. Venkatakrishnan and B. Tan \\ Additional information is available at the end of the chapter \\ http://dx.doi.org/10.5772/57101
}

\section{Introduction}

In general, nanocomposites are defined as the combination of multiphase materials in which at least one of the constituents has one dimension in the nanometer range [1]- [3]. The nanoscale constituent could be one dimensional likenanofibres and nanowires, two-dimensional like nanoclay or three-dimensional like spherical particles in nanoscale range. Nanofibers reinforced polymermultifunctionalitycan be attributed to the combination of the constituent materials. Desired properties of nanofibers reinforced polymercan be obtained by the selection of the constituent materials and the size of the nanofibress based on the required application. Current research has focused in the areas of manufacturing techniques and material combination for the fabrication of the nanostructured reinforced polymers [4], [5].

Nanofibers reinforced polymer are progressing with the use of a combination of atomic scale characterization and detailed modeling. In the early 1990s, Toyota Central Research Laboratories in Japan reported working on a Nylon-6 nanocomposite [6], in which a small amount of nano filler resulted in a considerable improvement of thermal and mechanical properties. The properties of nanofibers reinforced polymer materials depend on their morphology and interfacial characteristics as well as on the properties of their individual parents (nanofillers and polymer, in this case).

Dramatic changes in physical properties will be the result of the transition from microparticles to nanoparticles. Nanoscale materials have a large surface area for a given volume [7]. A nanostructured material can have substantially different properties from a larger-dimensional material of the same composition because many important chemical and physical interactions are governed by surfaces and surface properties. In the case of nanoparticles and nanofibers, the surface area per unit volume is inversely proportional to the material's diameter. So, the smaller the diameter, the greater is the surface area per unit volume [7]. Figure 1 shows 
common particle geometries and their respective surface area-to-volume ratios. For the nanofiber and layered material, the surface area to volume ratio is dominated by the first term in the equation, especially for nanomaterials. The second term $(2 / 1$ and $4 / 1)$ has a very small influence and is often omitted compared to the first term. Therefore, a changefrom the micrometer to nanometer in particle diameter, layer thickness, or fibrous material diameter range, will affect the surface area to volume ratio by three orders of magnitude [8].
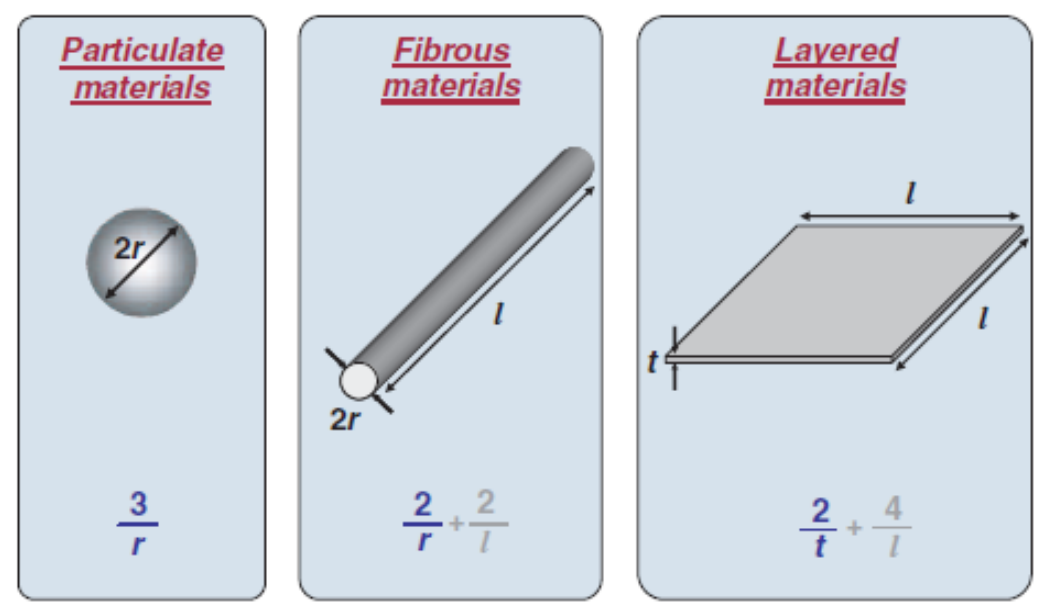

Figure 1. Common particle reinforcements/geometries and their respective surface areatovolume ratios. [8]

Typical nanomaterials currently under investigation include nanoparticles, nanotubes, nanofibers, fullerenes, and nanowires. In general, these materials are broadly classified by their geometries [9]: particle, layered, and fibrous nanomaterials [8], [9]. Carbon black, silica nanoparticle, polyhedral oligomericsislesquioxanes, can be classified as nanoparticle reinforcing agents, while nanofibers and carbon nanotubes are examples of fibrous materials [9]. When the filler has a nanometer thickness and a high aspect ratio (30-1000) plate-like structure, it is classified as a layered nanomaterial such as an organosilicate [10].

In general, the high aspect ratio of nanomaterials provides the necessary reinforcement properties. The properties of reinforced polymers are greatly influenced by the size of its nanomaterial and the quality of the interfacing between the matrix material and the filler material. Significant differences in composite properties may be obtained depending on the nature of the filler material used whether its layered silicate or nanofiber, cation exchange capacity, or polymer matrix and the method of preparation [11]. As an example, when the polymer is unable to intercalate (or penetrate) between the silicate sheets, a phase-separated composite is obtained, and the properties stay in the same range as those for traditional microcomposites [10]. In an intercalated structure, where a single extended polymer chain can penetrate between the silicate layers, a well-ordered multilayer morphology results with alternating polymeric and inorganic layers. An exfoliated or delaminated structure is obtained 
when the silicate layers are completely and uniformly dispersed in a continuous polymer matrix. In any case, the physical properties of the resultant nanofibers reinforced polymer will be significantly different, as discussed in the following sections. Similarly, in fibrous or particle-reinforced polymer nanofibers reinforced polymer (PNCs), dispersion of the nanoparticle and adhesion at the particle-matrix interface play crucial roles in determining the mechanical properties of the nanofibers reinforced polymer. The nanomaterial will not offer improved mechanical properties without proper dispersion. A poorly dispersed nanomaterial may degrade the mechanical properties of the produced reinforced polymers [12]. Additionally, optimizing the interfacial bond between the nanostructures and the matrix, one can tailor the properties of the overall nanofibers reinforced polymer in a similar manner to what is done in macrocomposites. As an example, good adhesion at the interface will improve properties such as interlaminar shear strength, fatigue, delamination resistance, and corrosion resistance. Finally, it is important to recognize that snanofibers reinforced polymer researches are extremely broad,encompassing areas such as communications, electronics and computing, data storage, aerospace and sporting materials, health and medicine, transportation, energy, environmental, and many other applications. The focus of this chapter is to highlight the state of knowledge in processing, fabrication, characterization, properties, and potential applications of the nanofibrous reinforced polymer microstructures.

\section{Fabrication techniques}

The advancement in the technology of MicroElectroMechanical Systems (MEMS) has demand for the fabrication of 3D micro/nanostructures and devices. The excitement surrounding the nanoscale science and technology gives us unique opportunities to develop and examine revolutionary processes and materials. Nanofibers reinforced polymer embedded with $2 \mathrm{D}$ and 3D micro/nano materials find many applications in the field of medicine, tissue engineering, drug delivery, antibacterial implants or catheters, modification of textiles, and modification of polymers. Many optical, electrical and magnetic applications, have opened up new areas of research for manufacturing nanofibers reinforced polymer with engineered nanoparticles materials.

Researchers have been working on different micro/nano manufacturing techniques. LIGA (German acronym for Lithographie, Galvanoformung, Abformung) [7], [8], Photolithography (6), Electrochemical Fabrication (EFAB) [9], localize electrochemical deposition [10] and laser sintering [13] are some of the techniques used for micro/nano fabrication. Some of these techniques have been used for the fabrication of nanofibers reinforced polymer by dispersing nanofibers in polymer resins.

The LIGA technique was invented approximately 20 years ago. It is a powerful method that facilitates the high volume production of nanofibers reinforced polymer components for many fields of applications [14]. Researchers presented designs, fabrication and experimental results of high power electrostatic microactuators, using LIGA process. This process is capable of producing high aspect ratio microstructures of nanofibers reinforced polymer, as shown in Figure 3 [15]. 
The LIGA process uses the simple shadow printing process onto a resist on an electrically conducting substrate. After development of the irradiated resist, an electroforming step fills the holes of the relief with metal. This more stable body is used as a mold insert for further molding or embossing steps [8]. The deep lithography step, performed by the use of highly parallel and collimated synchrotron radiation, is the basic step, thus not only defining the shape but also the structural accuracy of the final product. [16].

Typically LIGA structures allow for the free choice of the lateral 2D pattern that is projected into the third dimension to form prismatic or cylindrical geometries. Generally, this technique has been used to produce structures with straight walls. However, for all major LIGA process steps, variations have been developed to increase the fabrication flexibility. Geometrical variations in the third dimension (vertical) are possible and can be obtained in different ways by modifying or combining process steps, in particular for producing shapes with increased dimensionality. The consecutive process steps of deep UV lithography, electroforming, and plastic molding can be used to fabricate three dimensional (3D) microstructures with almost no restrictions in their lateral shape in a large variety of snanofibers reinforced polymer.

Photolithography is one of the widely used techniques for fabrication of nanofibers reinforced polymers. Two photolithography-based approaches has been presented to directly micromachinephotopatternablesuperhydrophobicmicropatterns with excellent adaptability and flexibility to a wide variety of substrates, employing the nanomorphology and hydrophobicity of polytetrafluoroethylene (PTFE) nanoparticles and the photopatternability and transparency of an SU-8 photoresist [17].

A light source such as UV light is used in photolithography to polymerize the photoresponsive resin with suspended nanoparticles through a mask [6]. Depending upon the requirement of the feature, a mask is prepared and the feature is transferred using a UV source light. The removal of the unaffected part by the light source is performed using the secondary process of chemical etching. UV light has been used primarily for transferring the mask, but various other alternatives such as X-ray lithography,lithography, nanoimprint and ion projection lithography are being explored [6], [18]. For several decades, this technique has been researched and developed, but the resolution of the fabricated feature using photolithography is limited due to the optical diffraction limitation. Photolithography also requires the use of expensive masks and molds for the fabrication process. These techniques are effective for the mass fabrication of high resolution microfeatures, but they are limited to 2D geometries [18]. Nanofibers reinforced polymer microstructures can be fabricated by dispersing the photosensitive resin with naomateirals [14]. Figure 2 shows SEM photographs of the photolithographic patterns for photosensitive polyimide with montmorillonitenanofibers reinforced polymers [19].

Electrochemical Fabrication (EFAB) was originally invited to addresses the long development time for Optical MEMS, which can go up to few weeks. This method has also been used for the fabrication of nanofibers reinforced polymer microstrucutres. The fabrication of nanofibers reinforced polymer film of polypyrrole (PPY) and $\mathrm{TiO}_{2}$ nanotube (TNT) arrays via electrochemical methods was reported in several articles. A novel dual-layered photore- 
ceptor based on the reinforced polymer film as a charge generation layer (CGL) was designed and fabricated [20].

EFAB is a solid free-form fabrication technology that creates complex, miniature threedimensional shapes based on 3-D computer aided design CAD data [8]. Inspired by rapid prototyping methods, EFAB can fabricate complex shapes by stacking multiple patterned layers. Unlike rapid prototyping, EFAB is a batch process that is suitable for volume production of fully functional devices in engineering materials, not only models and prototypes [9]. EFAB has some limitations and shortcomings. EFAB is a technique that requires the use of masks to build two-dimensional (2D) planar structures. High aspect ratio microstructures are thus a result of multiple steps of material deposition or removal through the use of several masks, that requires increased fabrication time and cost.

A conducting microelectrode is untilizedin a localized electrochemical deposition (LECD) technique to fabricate high aspect ratio structures. During fabrication, a localized deposition is produced by placing an electrode tip that has micrometre-scale dimensions, near a substrate in an electrolyte and applying an electric potential between them. Confined deposition is produced due to the highly localized electric field in the region between themicroelectrode and the substrate. High aspect ratio microstructures result from the displacement of the end of the electrode along the trajectory of the desired geometry while maintaining continuity with the deposited materials. However, nanocompositemicrostructures fabricated by this method are usually porous and have feature sizes in the tens of micrometers due to the limitation in fabricating and maintaining a sharp conductive probe, and in confining the electric field down to the nanoscale dimensions [10].

Laser sintering is another technique that has been widely used for the microfabrication of nanofibers reinforced polymers. In this method, a high intensity laser is used to ablate the material and form nanoparticles. Chen et al, reported a new technique for conductive nanofibers reinforced polymer microfabrication and they were able to lower the percolation threshold of the nanofibers reinforced polymer [21]. This process takes place inside a liquid polymer resin which is then polymerized using UV light to form nanofibers reinforced polymers layers. The main advantage of this technique is that metals, non-metals, glass, polymers can be utilized to fabricate nanofibers reinforced polymer s with various properties. The main drawback of this technique is its limitation to planar geometries. Stacking of different layers has been tried, but the alignment and handling of the layers contributes to the limitation of higher aspect ratio of the micro device [13].

The nonlinear optical process of multiphoton absorption (MPA) was first predicted in 1931 by the Nobel laureate physicist Marie Goeppert-Mayer in her doctoral dissertation [22]. The technique was not verified experimentally until the advent of laser. An intuitive explanation of MPA is the transition from the ground electronic state to an excited electronic state which is usually achieved by the absorption of a high-energy photon or instead reached by simultaneous absorption of multiple low energy photons. The most common implementation of this method is degenerate two-photon absorption (TPA) where both photons have the same energy. The spatial confinement of MPA excitation is used to induce a chemical reaction at the laser focal point. This polymerization reaction occurs by radical reaction mechanisms that 

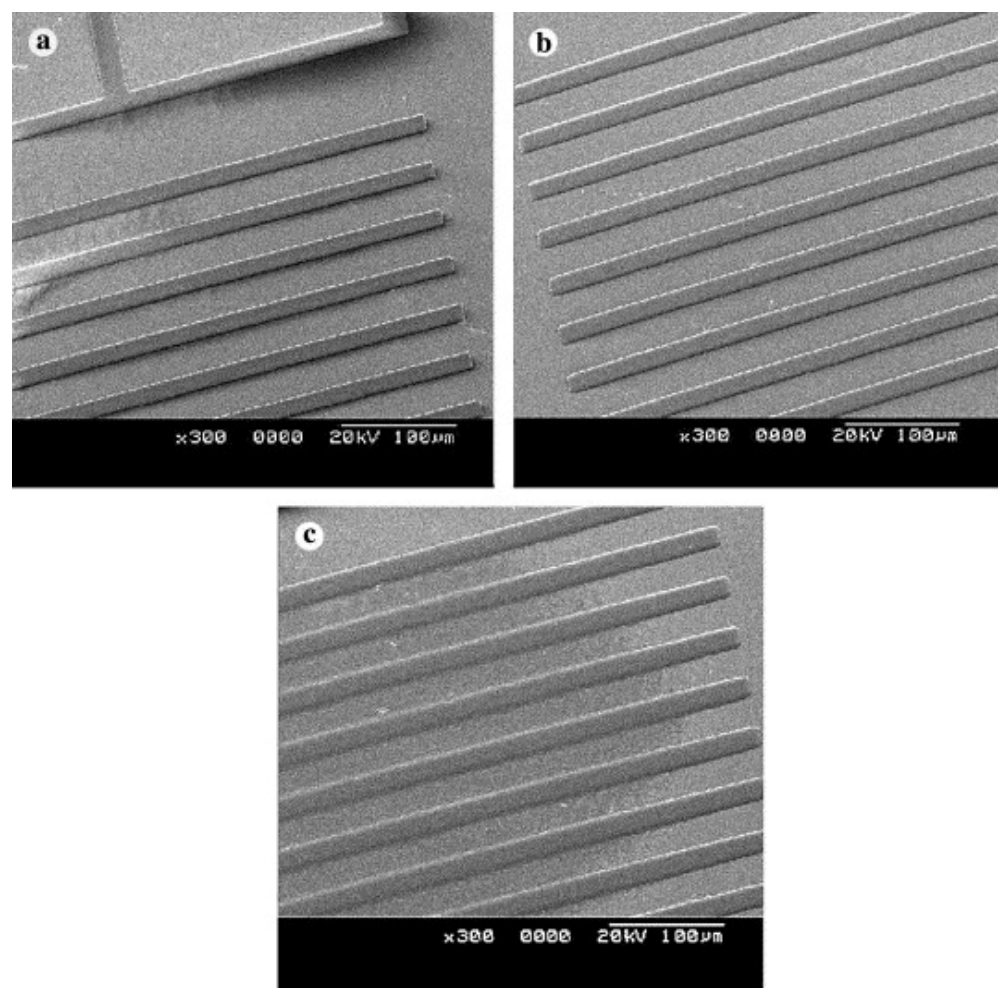

Figure 2. SEM photographs of the photolithographic patterns of (a) pure PSPI, PSPI/MMT nanofibers reinforced polymers with (b) 2 wt. \% and (c) 3 wt. \% MMT contents. [19].

depend on the photoinitiator and monomer being used. The photoinitiators used for radical MPA vary from small molecules to large conjugated molecules. A number of groups have reported the successful application of radical MPA using a different kind of resins, homemade and commercial, and different excitation sources. Custom photoinitiators have been designed by several groups and have been shown to be effective both for low threshold powers and for the ability to use less expensive laser systems. While the benefits of custom initiators are clear, their availability is limited. Commercial resins or resins made of commercial components have the advantage of accessibility but suffer from a slightly higher power threshold for fabrication. However, for the entire laser systems used, the threshold for these resins is always well below the available power and therefore their use is completely practical.

MPA can achieve resolution that is considerably better than that predicted by the diffraction limit due to a combination of optical nonlinearity. The probability for MPA is proportional to $I_{n}$, where $I$ is the light intensity and $n$ is the number of absorbed photons. This effectively narrows the point-spread function (PSF) of the beam near the focal point so that it is smaller than the diffraction limit at the excitation wavelength. The real benefit of the optical nonlinearity of MPA lies in the negligible absorption away from the focal point. Photoinitiator 


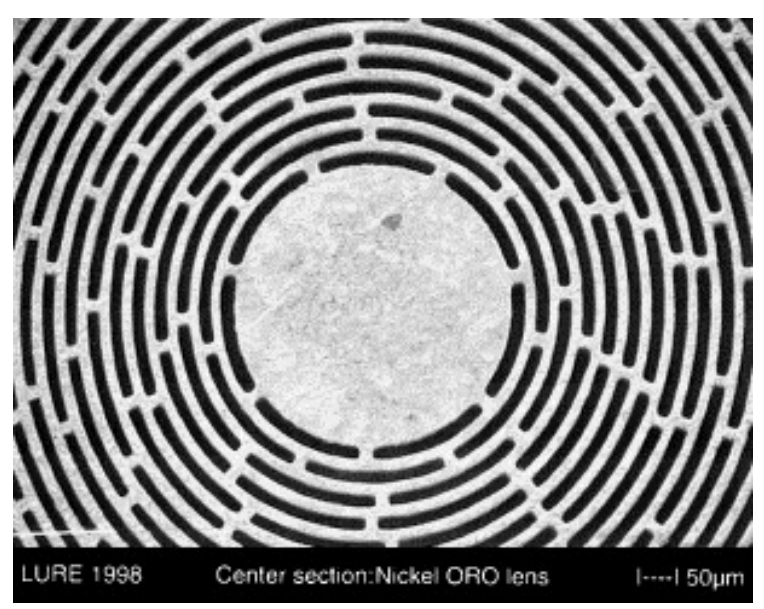

Figure 3. Center section of a fabricated ORO lens. Nickel matrix with $20 \mu \mathrm{m}$ wide and $600 \mu \mathrm{m}$ deep holes. Measured aspect ratios (sidewall inclination) $>400$ [15].

concentrations can be employed that are about ten times more than would be feasible for singlephoton excitation without any fear of out-of-plane polymerization.

Multi Photon Absorption (MPA) is a relatively new and evolving method for the manufacturing of micro/nano structures. Unlike other methods, this technique does not involve any secondary operations or processes for nanofibers reinforced polymer fabrication. Micro/nano structures and devices are fabricated with higher accuracy and complexity using MPA than other prevailing methods of fabrication for various applications. When an ultrashort laser pulse is focused in a photo responsive polymer resin, a solid voxel (volumetric pixel) is generated. The voxel size defines the minimum resolution of the polymer which is converted into solid form. Complex 2D and 3D microstructures can be fabricated by scanning the laser in the photo responsive resin [23]. Research on fabrication of micro/nano structures from different types of polymers using MPA has been conducted in the past decades. In certain applications there is a real need of complex nanofibers reinforced polymer structures fabrication [24]. The prevailing methods for nanofibers reinforced polymer manufacturing are good for planar or 2D device fabrication. For maskless manufacturing $2 \mathrm{D}$ and $3 \mathrm{D}$ nanofibers reinforced polymer devices with accuracy and precision there is a need of identifying or performance research towards the development of new maskless fabrication techniques using MPP due to its advantages over other methods.

\section{Nanofibers reinforced polymers enhanced properties}

The samples that presented in this section are polymer microstructures fabricated with MPA using laser ablative synthesis generated nanofibers as reinforcement [25] [26]. 


\subsection{Mechanical properties}

NanoIndentation Tester (Figure 4) uses an already established method where an indenter tip with a known geometry is driven into a specific site of the material to be tested, by applying an increasing normal load. When reaching a pre-set maximum value, the normal load is reduced until partial or complete relaxation occurs. At each stage of the experiment, the position of the indenter relative to the sample surface is precisely monitored with a differential capacitive sensor. For each loading/unloading cycle, the applied load value is plotted with respect to the corresponding position of the indenter. The resulting load/displacement curves provide data specific to the mechanical nature of the material under examination. Established models are used to calculatequantitative hardness and elastic modulus values for such data [27]. Hardness and elastic modulus can be determined using the method developed by Oliver and Pharr [28]. The hardness represents the resistance of a material to local surface deformation. The Indentation Testing Hardness, $\mathrm{H}$, is determined from the maximum load, $\mathrm{F}_{\max }$ divided by the projected contact area $A_{p}$ at the contact depth $h_{c}$ [29];

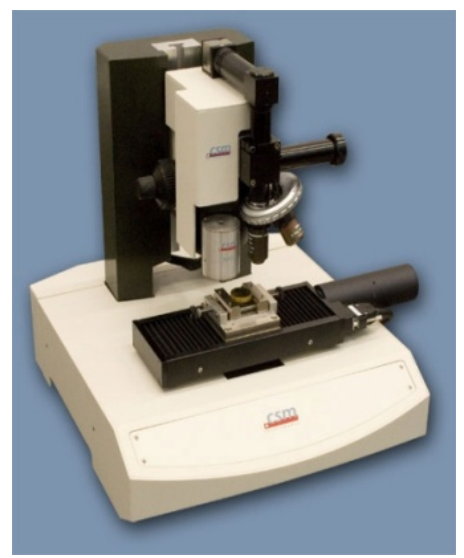

Figure 4. Nanolndentationtester (CSM Instruments)

$$
H=\frac{F_{\max }}{A_{p}\left(h_{c}\right)}
$$

Where $A_{p}\left(h_{c}\right)$ is the projected area of indenter contact at distance, $h_{c}$ from the tip and $h_{c}$ is the depth of the contact of the indenter with the test piece at $\mathrm{F}_{\max }$. Which can be expressed by $h_{c}=h_{\max }-\varepsilon\left(h_{\max }-h_{r}\right)$, where $\mathrm{h}_{\max }$ is the maximum indentation depth at $\mathrm{F}_{\max }, \mathrm{h}_{\mathrm{r}}$ is the point of intersection of the tangent to the unloading curve with the depth axis, and $\varepsilon$ is a constant depending on the nonlinearity of the unloading curve (Figure 5). For modified Berkovich indenter, $A_{p}\left(h_{c}\right)=24.5 h_{c}^{2}$ [30]. The elastic modulus represents the overall stiffness of the reinforced polymer network and the reduced modulus of the indentation contact, $\mathrm{E}_{\mathrm{r}}$, is given by [29]; 


$$
E_{r}=\frac{\sqrt{\pi} \cdot S}{2 \sqrt{A_{p}\left(h_{c}\right)}}
$$

where $\mathrm{S}$ is the contact stiffness or the slope of the unloading curve shown in Figure 5 at the point of maximum load which can be found by [31];

$$
S=\frac{F_{\max }}{h_{\max }-h_{r}}
$$

The relationship between Indentation Modulus, E, and the reduced modulus, $\mathrm{E}_{\mathrm{r}}$, of the sample is given by [29];

$$
\frac{1}{E_{r}}=\frac{1-v_{s}^{2}}{E}+\frac{1-v_{i}^{2}}{E_{i}}
$$

Where $v_{\mathrm{i}}$ is the Poisson's ratio of the indenter, $v_{\mathrm{s}}$ is the Poisson's ratio of the sample and $\mathrm{E}_{\mathrm{i}}$ is the modulus of the indenter. The elastic stress-strain characteristics of a randomly nanofibers dispersed polymer are expressed by three elastic constants, namely, Young's modulus E, Poisson's ratio $n$ vs, and shear modulus G. Only two of these three elastic constants are independent since they can be related by the following equation [32];

$$
G=\frac{E}{2\left(1+v_{s}\right)}
$$

By substituting the values of $\mathrm{E}$, and $v_{\mathrm{s}}$, the shear modulus can be calculated. A thin layer containing randomly oriented discontinuous nanofibers exhibits planar isotropic behavior. The properties are ideally the same in all directions in the plane of the layer. For such a layer, the tensile modulus and shear modulus are calculated from [32];

$$
\begin{aligned}
& E=\frac{3}{8} E_{11}+\frac{5}{8} E_{22} \\
& G=\frac{1}{8} E_{11}+\frac{1}{4} E_{22}
\end{aligned}
$$

where $E_{11}$ and $E_{22}$ are the longitudinal and transverse tensile moduli for a unidirectional discontinuous nanofiber lamina of the same nanofiber aspect ratio, and same nanofiber volume fraction as the randomly oriented discontinuous nanofiber composite. Rearrange Eqn 7 and subtract Eqn 6 from Eqn 7, then using transverse modulus $E_{22}=\frac{1+2 \eta_{t} v_{f}}{1-\eta_{t} v_{f}} E_{m}, \eta_{t}=\frac{\left(E_{f} / E_{m}\right)-1}{\left(E_{f} / E_{m}\right)+2}$, Poisson's ration $v_{s}=v_{f} v_{f}+v_{m} v_{m}$ and $v_{f}+v_{m}=1$, the volume fraction ratio of the nanofibers in the polymer matrix can be obtained by [31]; 


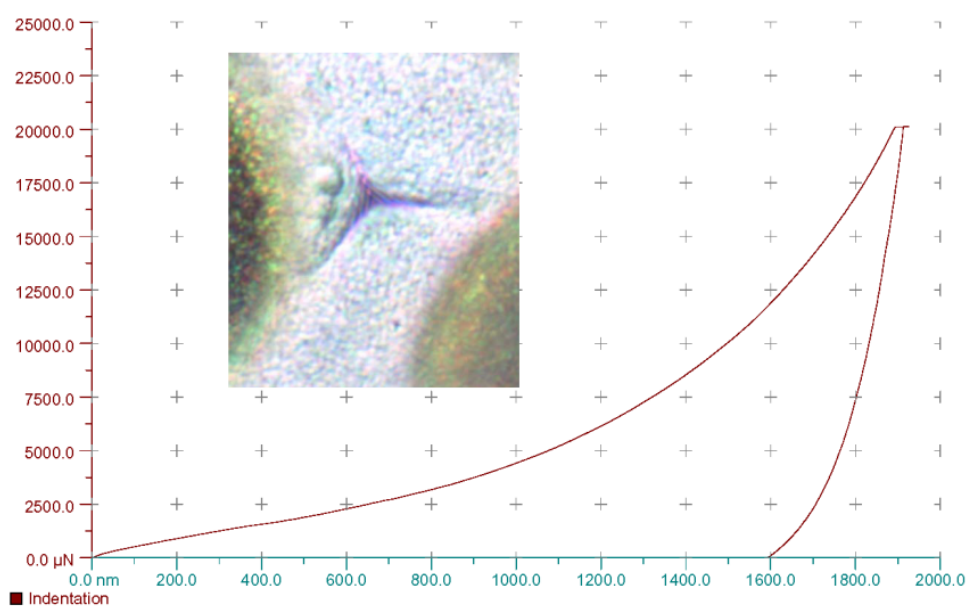

Figure 5. Typical indentation load-displacement curves for the reinforced polymer

$$
v_{f}=\frac{\frac{8(3 G-E)}{E_{m}}-1}{\left(\frac{\left.E_{f} / E_{m}\right)-1}{\left(E_{f} / E_{m}\right)+2}\right)\left(2+\frac{8(3 G-E)}{E_{m}}\right)}
$$

Where $v_{\mathrm{m}}$ is the volume fraction of the polymer matrix, $v_{\mathrm{f}}$ and $v_{\mathrm{m}}$ are Poisson's rations of nanofibers and polymer matrix respectively, $E_{f}$ and $E_{m}$ are Young's moduli for nanofibers and polymer matrix respectively. By substituting the appropriate values in Eqn 8, the volume fraction of the nanofibers in the polymer matrix can be calculated. An important function of the matrix in a nanofiber-reinforced composite material is to provide lateral support and stability for nanofibers under longitudinal compressive loading like the one applied by the indenter head. In polymer matrix composites with which the matrix modulus is relatively low compared to the nanofiber modulus, failure in longitudinal compression is usually initiated by localized buckling of nanofibers. In general, the shear mode of failure is more important than the extensional mode of failure and the shear mode is usually controlled by the matrix shear modulus as well as nanofiber volume fraction.

A viscoelastic polymer has both elastic component and a viscous component. Applying stress to a polymer causes molecular rearrangement due to changing positions by parts of the long polymer chain (creep). Polymers remain solid even when these parts of their chains are rearranging in order to accompany the stress which creates a back stress in the material. The material no longer creeps when the back stress is of the same magnitude as the applied stress. If the original stress is taken away, the accumulated back stresses will cause the polymer to get back to its original form with the help of its elastic component. The polymer loses energy when a load is applied then removed with the area between loading and unloading curves (Figure 5) being equal to the energy lost during the loading cycle. Thus permanent plastic deformation of the polymer occurs even when the applied load is removed. 
The large surface area of nanofibers provides better interaction between the polymer chains and nanofibers. The nanofibrous structures act as a preferential nucleation site for crystalline phases which cause an increase in the modulus and hardness. Hardness, which is directly related to the flow strength of a material, depends on the effective load transfer between the matrix and the reinforcement phase in the nanofibers reinforced polymer. Strong interfacial bonding between the matrix and the nanofibers is essential for efficient load transfer to obtain high strength. Plastic deformation in polymers occurs by nucleation and propagation of shear bands which in the unreinforced polymer matrix propagate unhindered as there are no barriers for their movement. Thus, the presence of nanofibers in the nanofibers reinforced polymers could offer resistance for the propagation of shear bands. A good mechanical interlocking and the presence of obstacles to the motion of shear bands are the reasons for the enhancement of hardness and elastic modulus in polymer dispersed nanofibers.

\subsubsection{Electrical conductivity}

Many techniques for the synthesis of conductive polymers have been developed. Most conductive polymers are prepared by oxidative coupling of monocyclic precursors. One challenge is usually the low solubility of the polymer. An important property of the electrically conductive reinforced polymer is the positive temperature coefficient (PTC) effect. PTC reflects the increase in electrical resistivity of the composites during the heating process, and hence decreasing the electrical conductivity of the reinforced polymer. PTC materials have so many potential applications, including sensors, self-regulating heaters, and switching materials. The PTC behavioroccurs as a result of the difference in the coefficients of thermal expansion between the fillers and the matrix.

Electrical properties of compositions made of nanofibers, dispersed in polymers, are mainly characterized by the formation of a conduction network by contact conditions between neighboring nanofibers in the network. Carbon nanofibers (CFN's) dispersed in the developed resin was used as a conductive composition materials in several applications. The conductivity of the nanofibers reinforced polymer films can be measured using two points probe testing device from SVSLabsInc as shown in Figure 6. Four probe systems can also be used. The voltage passing through the nanofibers reinforced polymer film can be recorded, as well as the current and the value of the resistance (and hence the conductivity) can be obtained using Ohm's law.

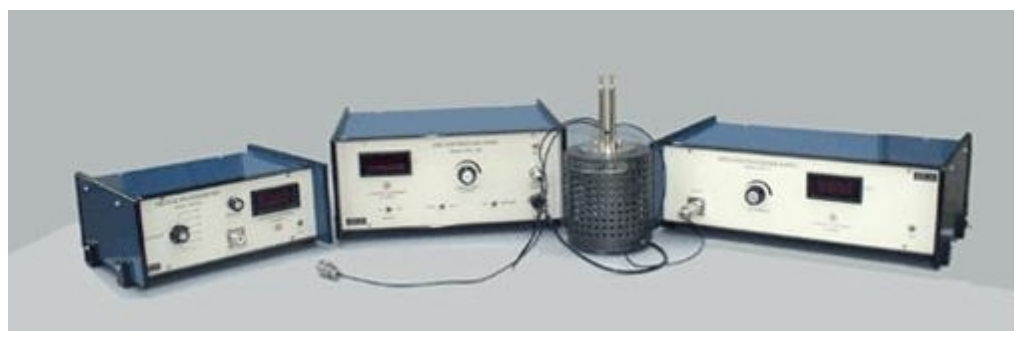

Figure 6. Two points probe testing device (SVSLabsInc). 
Electrical conductivity in CNF's reinforced polymer is generated as a result of the direct contact between CNF's and tunneling resistance determined by the width of the insulating resin around the CNF's. Thermal expansion caused by heat gradient increases gap width between contiguous CNF's and reduces the number of conductive pathways which results in a decrease in electrical conductivity. The temperature dependence of the electrical conductivity can be explained by the general theory of the thermal fluctuations. The resistivity of the junction is given by;

$$
\rho=\rho_{o} e^{-T_{1} /\left(T+T_{0}\right)}
$$

Where the constants $Q_{0}, T_{1}$, and $T_{0}$ depend essentially on the characteristics of the tunnel junctions, which are supposed to be functions of various parameters such as filling factor, filler size and shape, sample processing. The relative resistivity $\left(\varrho_{\mathrm{r}}\right)$ can be used to characterize the intensity of the PTC effect:

$$
\rho_{r}=\log \left(\frac{\rho_{140}}{\rho_{20}}\right)
$$

Where $Q_{140}$ and $\varrho_{20}$ are the resistances of the composites at two different temperatures, for example, 140 and $20^{\circ} \mathrm{C}$. Thus the relative conductivity can be expressed as;

$$
S_{r}=\log \left(\frac{\rho_{20}}{\rho_{140}}\right)
$$

This represents how sensitive and to what extent the reinforced polymer resistance responds after being stimulated by the temperature change. The electrical sensitivity of a reinforced polymer fabricated by femtosecond laser material processing can be expressed by;

$$
K_{S}=\frac{\Delta S_{r}}{\Delta \omega_{r}}
$$

Where $\mathrm{K}_{\mathrm{s}}$ is the electrical sensitivity, $\Delta \mathrm{S}_{\mathrm{r}}$ is the change in relative conductivity and $\Delta \omega_{\mathrm{r}}$ is the change in relative repetition rate of the femtosecond laser that can be expressed by;

$$
\omega_{r}=\frac{\omega}{\omega_{\min }}
$$

Where $\omega_{\min }$ is the minimum repetition rate used for the generation of nanofibers. For example, The equation of the straight line obtained from Figure 7 is $S_{r}=0.17 \omega_{r}-10.6117$ which indicate that the electrical sensitivity $\mathrm{K}_{\mathrm{s}}$ is about 0.17 . The higher the value of the electrical sensitivity, the more sensitive is the reinforced polymer to change in temperature and thus less conductivity at higher temperatures. Also, the PTC effect of nanofibers reinforced polymers fabricated with higher laser repetition rate is weaker than that of nanofibers reinforced polymer $\mathrm{s}$ 
fabricated with lower repetition rate. This is a direct result of the matrix volumetric expansionwhich cause more contiguous CFN's to get disconnected and reduce number of electrical pathways.

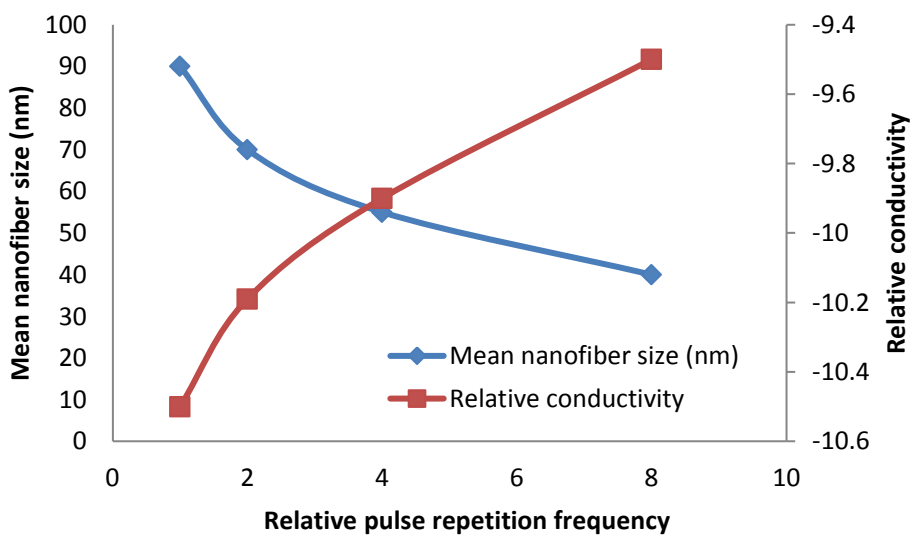

Figure 7. Pulse repetition frequency effect on the relative conductivity of the nanofibers reinforced polymer microstructures.

\subsubsection{Magnetic enhancement}

Magnetic neodymium-iron-boron $(\mathrm{NdFeB})$ nanofibers and nanoparticles, have become one of the most important spot in the research field of magnetic nanomaterials to meet the demand for miniaturization of electronic components recently. They have been successfully prepared by various techniques like the sol-gel auto-combustion method [33], co-precipitation [34], hydrothermal method [35], reverse micelles [36], microemulsion method [37], alternate sputtering [38], pulsed laser deposition [39], and many others.

The total magnetization of a nanofiber is given by the vectorial sum of all single magnetic moments of the atoms. As for the atomic magnetic moments in nanofibers, the average magnetization will be zero in the absence of magnetic field since all magnetic moments are randomly directed in space. When a magnetic field is applied by the substrate, the magnetic moments orient in the direction of the field and give rise to a net magnetization of the nanofibers [40].

In ferromagnetic materials (Figure 8 (a) ), the saturation magnetization MS of the magnetic nanofibers reinforced polymer microstructures at room temperature is higher than that of the pure polymer. The larger coercivitiesHC that we see could be as a result of the oxide Fe. Figure 8 (b) shows the room temperature M-H curves for the polymer (Ormocer). It has a weak diamagnetic response to the applied field, but appears to have a small amount of unknown impurity which contributes to a very weak non- linear deviation near zero fields that could be a paramagnetic effect. Small traces of paramagnetic impurities can be found in almost all 
materials and hence, it is not surprising in these polymers. However, these trace impurities do not affect the quality of our ferromagnetic composites. Figure 8 (c) shows the magnetic measurements of the nanofibers reinforced polymers which has a coercive field $\mathrm{HC}$ of approximately 260 Oe at room temperature. This large coercivity suggests that oxide nanofibrers are present on the microstructure.

It can be seen that the magnetization of the generated nanofibers reinforced polymer increases compared to pure polymer. This increase in magnetization is expected below the superparamagnetic- ferromagnetic transition temperature, which is above $300 \mathrm{~K}$ for the magnetic nanofibers of $20 \mathrm{~nm}$ average size due to reduced thermal activation energy. Nanoparticle interactions, which depend on the iron concentration in the polymer matrix, strongly influence the remnant magnetization. Since agglomeration of nanoparticles into nanofibers is observed in all our polymer nanofibers reinforced polymer samples, interactions are expected to play a significant role in the magnetic response. These interactions lead to a non- linear increase in $\mathrm{MR}$ as the concentration of iron is increased. The magnetic interactions are generally expected to be dipolar in nature, although in strongly coupled clusters, exchange interactions are also possible.

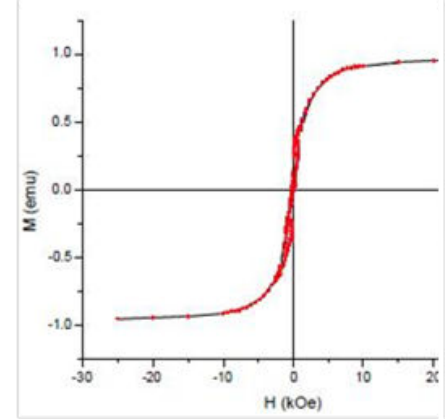

(a)

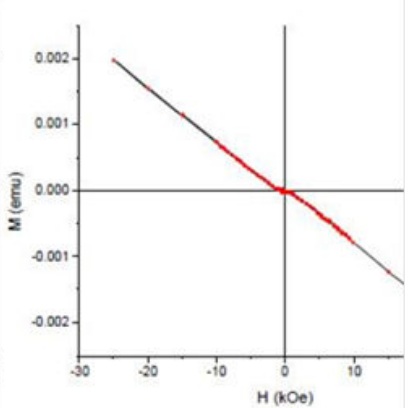

(b)

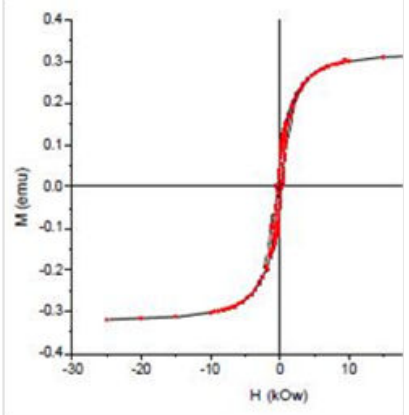

(c)

Figure 8. Room temperature M-H curves (a) Magnetic nanofiberous structures, (b) Ormocer, and (c) magnetic nanofibers reinforced polymer.

\section{Applications}

\subsection{Bioapplications}

There are unlimited applications of reinforced polymer microstructures. One of the most recent application is DNA sensing based on reinforced polymer microstructuring. A conducting polymer containing nanofibrous structure can be very sensitive even to small perturbations at the interface, thanks to its high electrical conductivity and electron transfer capabilities when the interface is covered by its film. Biocompatible conducting polymers can be variably 
modified for immobilization of probe DNA via covalent linking or electrostatic interactions. The conductivity of the conducting polymer electrochemically deposited on the electrode surface can be modulated by changing the $\mathrm{pH}$ of the medium, the electrochemical potential, and/or the electrolyte. Because of these characteristics and other advantages, conducting polymers could be utilized extensively for the construction of biosensors including DNA sensors which, to the best of our knowledge, is reported for the first time.

Noble metals are known to create strong chemical bonds with compounds terminating with a thiol (-SH) group. In order to achieve immobilization of nucleic acids on solid substrates, researchers have developed techniques by which DNA molecules can be linked to a thiol group. In this case, the thiolated terminal of molecules is chemisorbed on the substrate, while the DNA portion of the molecules are standing parallel to each other and away from the substrate. Since they are formed quickly, resulting in a well-defined and reproducible surface, and are stable under normal laboratory conditions, gold electrodes are fabricated using femtosecond laser material processing or other techniques [41].

\subsection{Energy applications}

Reinfoced polymer microstructuring can be utilized in neomerous application. Researchers prepared dye-sensitized solar cells using micro/nanofibers reinforced polymer $\mathrm{TiO}_{2}$ porous films [42]. This result in cells with enhanced light collection. They applied a technique which opens an alternative way for manufacturing solar cells on an industrial scale. $\mathrm{Ti}_{2}$ micro/nanocomposite structured electrodes for quasi-solid-state dye-sensitized solar cells [43]. These revolutionary nano-structured ultra thin film solar PV products will provide affordable clean renewable energy for everyone. Another unique technology has been developed that absorbs and converts more sunlight throughout the day by utilizing special kind of nanofibers reinforced polymers. This result in a dramatic increase in total power output. Each nanofiber increases the total PV surface area by an incredible 6-12 times over current other thin film products on the market today. Figure 9 shows luminescent solar concentrators (LSCs) comprising CdSe core/multishell quantum dots (QDs) developed by Bomm et al [44].
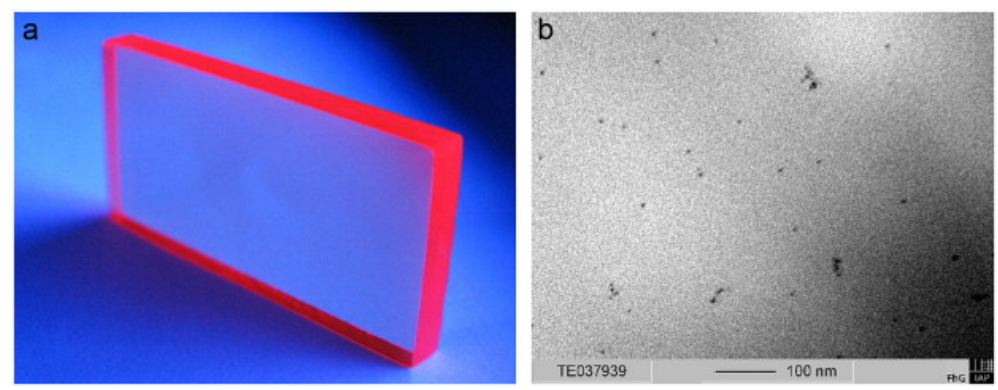

Figure 9. (a) Photograph of a P (LMA-CO-EGDM) plate containing CdSe core/multishell QDs (illuminated by a UV-lamp) illustrating the concentrator effect and (b) TEM image of a QD-LSC/P (LMA-Co-EGDM) nanofibers reinforced polymer showing single QDs and a few small QD aggregates [44]. 


\section{Summary}

In conclusion, this chapter focused on nanofibers reinforced polymer microstructures, including fundamental properties, manufacturing techniques, and applications. The chapter also discussed the scientific principles and mechanisms in relation to the methods of processing, manufacturing and commercial applications. The mechanical, electrical, and magnetic properties of nanofibers reinforced polymers has been discussed in details and it offers insight studies on technology, modeling, characterization, processing, manufacturing, and applications for nanofibers reinforced polymer nanocomposites.

\section{Author details}

A. Alubaidy ${ }^{1}$, K. Venkatakrishnan ${ }^{2}$ and B. $\operatorname{Tan}^{3 *}$

*Address all correspondence to: tanbo@ryerson. ca

1 School of Mechanical and Electrical Engineering, Sheridan Institute of Technology \& Advanced Learning, Brampton, Canada

2 Department of Mechanical Engineering, Ryerson University, Toronto, Ontario, Canada

3 Department of Aerospace Engineering, Ryerson University, Toronto, Ontario, Canada

This chapter is designed to be a comprehensive source for nanofibers reinforced polymer studies. The fundamental properties, manufacturing techniques, and applications of nanofibers reinforced polymer materials are discussed. In addition, this chapter introduce an in depth scientific framework for the advances in nanofibers reinforced polymer researches as well as scientific principles and mechanisms in relation to the methods of fabrication of reinforced microstructuring with a discussion on potential commercial applications. The mechanical, electrical, and magnetic properties of nanofibers reinforced polymer microstructures will be the focus of this chapter. It also offers an in depth discussion on methodology, modeling, characterization, fabrication, and applications for nanofibers reinforced polymers.

\section{References}

[1] T. Rogers-Hayden and N. Pidgeon, “Moving engagement 'upstream'? Nanotechnologies and the Royal Society and Royal Academy of Engineering's inquiry," Public Understanding of Science, vol. 16, no. 3, pp. 345-364, Jul. 2007. 
[2] A. Pomogailo, "Synthesis and intercalation chemistry of hybrid organo-inorganic nanocomposites," Polymer Science Series C, vol. 48, no. 1, pp. 85-111, 2006.

[3] J. -J. Luo and I. M. Daniel, "Characterization and modeling of mechanical behavior of polymer/clay nanocomposites," Composites Science and Technology, vol. 63, no. 11, pp. 1607-1616, Aug. 2003.

[4] E. Thostenson, C. Li, and T. Chou, "Nanocomposites in context," Composites Science and Technology, vol. 65, no. 3-4, pp. 491-516, Mar. 2005.

[5] M. Alexandre and P. Dubois, "Polymer-layered silicate nanocomposites: preparation, properties and uses of a new class of materials," Materials Science and Engineering: $R$ : Reports, vol. 28, no. 1-2, pp. 1-63, Jun. 2000.

[6] J. Li and L. S. Melvin, "Sub-resolution Assist Feature Modeling for Modern Photolithography Process Simulation," Japanese Journal of Applied Physics, vol. 47, p. 4862, 2008.

[7] A. Bertsch, H. Lorenz, and P. Renaud, "Combining microstereolithography and thick resist UV lithography for 3D microfabrication," in Micro Electro Mechanical Systems, 1998. MEMS 98. Proceedings., The Eleventh Annual International Workshop on, 1998, pp. 18-23.

[8] A. Schmidt and W. Ehrfeld, "Recent developments in deep x-ray lithography," Journal of Vacuum ScienceETechnology B: Microelectronics and Nanometer Structures, vol. 16, no. 6, pp. 3526-3534, Jan. 1998.

[9] A. Cohen, G. Zhang, F. G. Tseng, U. Frodis, F. Mansfeld, and P. Will, “EFAB: rapid, low-cost desktop micromachining of high aspect ratio true 3-D MEMS," in Micro Electro Mechanical Systems, 1999. MEMS'99. Twelfth IEEE International Conference on, 1999, pp. 244-251.

[10] J. D. Madden and I. W. Hunter, "Three-dimensional microfabrication by localized electrochemical deposition," Microelectromechanical Systems, Journal of, vol. 5, no. 1, pp. 24-32, 1996.

[11] S. Barcikowski, M. HUSTEDT, and B. CHICHKOV, "Nanocomposite manufacturing using ultrashort-pulsed laser ablation in solvents and monomers," Polimery, vol. 53, no. 9, pp. 657-662, 2008.

[12] Z. B. Sun, X. Z. Dong, S. Nakanishi, W. Q. Chen, X. M. Duan, and S. Kawata, “Logpile photonic crystal of CdS-polymer nanocomposites fabricated by combination of two-photon polymerization and in situ synthesis," Applied Physics A: Materials Science E Processing, vol. 86, no. 4, pp. 427-431, 2007.

[13] X. -Z. D. Z. -B. Sun, "Log-pile photonic crystal of CdS-polymer nanocomposites fabricated by combination of two-photon polymerization and in situ synthesis," vol. 86, no. 4, pp. 427-431, 2007. 
[14] C. K. Malek and V. Saile, "Applications of LIGA technology to precision manufacturing of high-aspect-ratio micro-components and-systems: a review," Microelectronics Journal, vol. 35, no. 2, pp. 131-143, 2004.

[15] R. K. Kupka, F. Bouamrane, C. Cremers, and S. Megtert, "Microfabrication: LIGA-X and applications," Applied Surface Science, vol. 164, no. 1-4, pp. 97-110, Sep. 2000.

[16] R. Kondo, S. Takimoto, K. Suzuki, and S. Sugiyama, "High aspect ratio electrostatic micro actuators using LIGA process," Microsystem technologies, vol. 6, no. 6, pp. 218221, 2000 .

[17] L. Hong and T. Pan, "PhotopatternableSuperhydrophobicNanocomposites for Microfabrication," Journal of Microelectromechanical Systems, vol. 19, no. 2, pp. 246-253, Apr. 2010.

[18] X. Zhang and C. Sun, "Experimental and numerical investigations on microstereolithography of ceramics," Journal of Applied Physics, vol. 92, no. 8, p. 4796, Jan. 2002.

[19] J. Marqués-Hueso, R. Abargues, J. L. Valdés, and J. P. Martínez-Pastor, “Ag and Au/ DNQ-novolacnanocompositespatternable by ultraviolet lithography: a fast route to plasmonic sensor microfabrication," J. Mater. Chem., vol. 20, no. 35, pp. 7436-7443, 2010.

[20] M. Ouyang, R. Bai, Y. Xu, C. Zhang, C. A. Ma, M. Wang, and H. Z. Chen, “Fabrication of polypyrrole/TiO2 nanocomposite via electrochemical process and its photoconductivity," Transactions of Nonferrous Metals Society of China, vol. 19, no. 6, pp. 1572-1577, 2009.

[21] D. Z. Chen, S. Lao, J. H. Koo, M. Londa, and Z. Alabdullatif, “Powder Processing and Properties Characterization of Polyamide 11-Graphene anocomposites for Selective Laser Sintering," in Proc. 2010 solid freeform fabrication symposium, Austin, TX, August, 2010, pp. 2-4.

[22] M. Goeppert-Mayer, "ON ELEMENTARY ACTS WITH TWO QUANTUM JUMPS.," May 1967.

[23] T. Baldacchini, "Acrylic-based resin with favorable properties for three-dimensional two-photon polymerization," Journal of Applied Physics, vol. 95, no. 11, p. 6072, Jan. 2004.

[24] K. -S. Lee, D. -Y. Yang, S. H. Park, and R. H. Kim, "Recent developments in the use of two-photon polymerization in precise 2D and 3D microfabrications," Polymers for Advanced Technologies, vol. 17, no. 2, pp. 72-82, 2006.

[25] M. Alubaidy, K. Venkatakrishnan, and B. Tan, "Fabrication of a reinforced polymer microstructure using femtosecond laser material processing," J. Micromech. Microeng., vol. 20, no. 5, p. 055012, May 2010. 
[26] M. Alubaidy, B. Tan, A. Mahmood, and K. Venkatakrishnan, "Nanofiber Plasmon Enhancement of Two-Photon Polymerization Induced by Femtosecond Laser," J. Nanotechnol. Eng. Med., vol. 1, no. 4, pp. 041015-041015, Nov. 2010.

[27] B. Gu and W. Ji, "Two-step four-photon absorption," Opt Express, vol. 16, no. 14, pp. 10208-10213, Jul. 2008.

[28] W. c. Oliver and G. m. Pharr, "An improved technique for determining hardness and elastic modulus using load and displacement sensing indentation experiments," Journal of Materials Research, vol. 7, no. 06, pp. 1564-1583, 1992.

[29] B. Bhushan and X. Li, "Micromechanical and tribological characterization of doped single-crystal silicon and polysilicon films for microelectromechanical systems devices," Journal of Materials Research, vol. 12, no. 01, pp. 54-63, 1997.

[30] S. Carusotto, G. Fornaca, and E. Polacco, "Multiphoton Absorption and Coherence," Phys. Rev., vol. 165, no. 5, pp. 1391-1398, Jan. 1968.

[31] M. Alubaidy, B. Tan, A. Mahmood, and K. Venkatakrishnan, “Mechanical Property Enhancement of Nanocomposite Microstructures Generated by Two Photon Polymerization," J. Nanotechnol. Eng. Med., vol. 1, no. 4, pp. 041016-041016, Nov. 2010.

[32] P. K. Mallick, Fiber-Reinforced Composites: Materials, Manufacturing, and Design. M. Dekker, 1993.

[33] N. T. K. Thanh, Magnetic Nanoparticles: From Fabrication to Clinical Applications. CRC Press, 2012.

[34] P. Kruus, M. O'Neill, and D. Robertson, “Ultrasonic initiation of polymerization," Ultrasonics, vol. 28, no. 5, pp. 304-309, Sep. 1990.

[35] G. J. Lee, S. H. Lee, K. S. Ahn, and K. H. Kim, "Synthesis and characterization of soluble polypyrrole with improved electrical conductivity," Journal of Applied Polymer Science, vol. 84, no. 14, pp. 2583-2590, 2002.

[36] J. R. Li, J. R. Xu, M. Q. Zhang, and M. Z. Rong, “Carbon black/polystyrene composites as candidates for gas sensing materials," Carbon, vol. 41, no. 12, pp. 2353-2360, 2003.

[37] J. X. Li, M. Silverstein, A. Hiltner, and E. Baer, "The ductile-to-quasi-brittle transition of particulate-filled thermoplastic polyester," Journal of Applied Polymer Science, vol. 52, no. 2, pp. 255-267, 1994.

[38] S. Loshaek, "Crosslinked polymers. II. Glass temperatures of copolymers of methyl methacrylate and glycol dimethacrylates," Journal of Polymer Science, vol. 15, no. 80, pp. 391-404, Feb. 1955.

[39] K. Matyjaszewski, Cationic Polymerizations: Mechanisms, Synthesis E Applications. Taylor \& Francis, 1996. 
[40] M. -A. Alubaidy, K. Venkatakrishnan, and B. Tan, "Synthesis of magnetic nanofibers using femtosecond laser material processing in air," Nanoscale Research Letters, vol. 6, no. 1, p. 375, May 2011.

[41] M. Alubaidy, L. Soleymani, K. Venkatakrishnan, and B. Tan, "Femtosecond laser nanostructuring for femtosensitive DNA detection," Biosensors and Bioelectronics, vol. 33, no. 1, pp. 82-87, Mar. 2012.

[42] A. S. Mahmood, K. Venkatakrishnan, B. Tan, and M. Alubiady, "Effect of laser parameters and assist gas on spectral response of silicon fibrous nanostructure," Journal of Applied Physics, vol. 108, no. 9, pp. 094327-094327-6, 2010.

[43] Y. Zhao, J. Zhai, S. Tan, L. Wang, L. Jiang, and D. Zhu, “TiO2 micro/nano-composite structured electrodes for quasi-solid-state dye-sensitized solar cells," Nanotechnology, vol. 17, p. 2090, 2006.

[44] J. Bomm, A. Büchtemann, A. J. Chatten, R. Bose, D. J. Farrell, N. L. A. Chan, Y. Xiao, L. H. Slooff, T. Meyer, A. Meyer, W. G. J. H. M. van Sark, and R. Koole, "Fabrication and full characterization of state-of-the-art quantum dot luminescent solar concentrators," Solar Energy Materials and Solar Cells, vol. 95, no. 8, pp. 2087-2094, Aug. 2011. 